math-ph/0111011

\title{
The Combinatorics of Alternating Tangles: from theory to computerized enumeration
}

\author{
J. L. Jacobsen and P. Zinn-Justin \\ Laboratoire de Physique Théorique et Modèles Statistiques \\ Université Paris-Sud, Bâtiment 100 \\ 91405 Orsay Cedex, France
}

\begin{abstract}
We study the enumeration of alternating links and tangles, considered up to topological (flype) equivalences. A weight $n$ is given to each connected component, and in particular the limit $n \rightarrow 0$ yields information about (alternating) knots. Using a finite renormalization scheme for an associated matrix model, we first reduce the task to that of enumerating planar tetravalent diagrams with two types of vertices (self-intersections and tangencies), where now the subtle issue of topological equivalences has been eliminated. The number of such diagrams with $p$ vertices scales as $12^{p}$ for $p \rightarrow \infty$. We next show how to efficiently enumerate these diagrams (in time $\sim 2.7^{p}$ ) by using a transfer matrix method. We give results for various generating functions up to 22 crossings. We then comment on their large-order asymptotic behavior.
\end{abstract}

$10 / 2000$ 


\section{Introduction}

The mathematical description of knots and related combinatorial objects is a subject with a history of several centuries. Giving an account of the progress made over the years is beyond the scope of the present paper, and we refer to [1] for an introduction to the existing literature (from the 19th century onwards). An old idea, first published by Ozanam [2] in 1694, is to treat a knot as a planar diagram with over- and underpasses. This trick, however simple it may appear, is essential to the present work, since it allows us to effectively reduce the dimension of the problem from three to two, and in particular to apply the powerful tools of matrix models in the planar limit. The classification of such diagrams with respect to topological equivalences calls for a particular geometrical framework (tagged geometria situs by Leibniz [3]), as was probably first pointed out by Vandermonde [4] in 1771.

For technical reasons, we shall here constrain our attention to the subset of alternating knots (and various generalizations such as links and tangles), which by definition possess a representation as a diagram in which over- and underpasses alternate. The first efforts in this domain date from the late 19th century through the pioneering works of Tait, Kirkman and Little. Tait originally thought that all knots were alternating, but Little made it evident (though no proof was known at that time) that non-alternating knots start appearing at order $p=8$ intersections. It is now believed that alternating knots are asymptotically subdominant (the corresponding statement has been proven rigorously in the case of links [5]). However, the enumeration of alternating knots remains a very appealing mathematical problem, and to this date no explicit formula giving the number of knots at order $p$ is known.

The subtle question of factoring out topological equivalences for alternating knots is facilitated by two remarkable theorems which were first conjectured by Tait, but only proved rigorously quite recently. First, alternating diagrams can be reduced to diagrams having minimal crossing number by iteratively eliminating irrelevant crossings through the move shown in Fig. 3 a [6]. Second, two such reduced alternating diagrams are topologically equivalent if and only if they are connected by a sequence of simple transformations (see Fig. 4) known as flypes [7].

In the first part of the paper we shall show that counting alternating knots modulo the flype equivalence is equivalent to computing the generating function for a certain class of planar tetravalent diagrams that are illustrated in Fig. 2. These diagrams possess two 
types of vertices (self-intersections and tangencies), which are weighted separately. The proof is inspired by the renormalization procedure originally invented within the context of quantum field theory (QFT), and here applied to the random matrix model that generates the above-mentioned planar diagrams. In particular we show that factoring out topological equivalences is tantamount to including various counterterms in the effective action of the matrix model. Although we have found it appropriate to state the argument using the language of physics, it should be stressed that it eventually involves nothing but formal manipulations of generating functions, and thus is completely rigorous.

Unfortunately we have not found a way of analytically computing the generating function of the diagrams depicted in Fig. 2 for general $n$, although a number of cases $(n=1$ [5], $n=2$ [8], $n=\infty$ [9] and $n=-2$ [10]) can be solved exactly. In the second part of the paper we therefore proceed to describe how the integer coefficients of the generating function can be found, up to any desired order $p$, using a computer. The number of diagrams to be enumerated scales as $12^{p}$ for $p \gg 1$, but our algorithm does the counting in a most expeditious fashion, only using a time $\sim 2.7^{p}$. Once again, we have inspired ourselves from theoretical physics, reformulating the counting process as the action of a transfer matrix, which is an object normally used to describe the discrete time evolution of a quantum system. Mathematicians may think of the transfer matrix as a linear operator that acts on a set of basis states consisting of appropriately defined sub-diagrams.

From the description given below it will be evident that the applicability of our transfer matrix method extends far beyond the context of the present paper. Indeed, it can be generalized to the computation of generating functions for all sorts of planar diagrams, provided that the latter have at least one external leg. In particular, we are presently unable to generate results for closed knots (usually known in the mathematics literature simply as knots, as opposed to tangles which possess external legs).

We finally state our results, here given up to a maximal order of $p=22$, and we analyze the asymptotic behavior of the various generating functions. The number of objects at order $p \gg 1$ is generally supposed to have the asymptotic behavior $c \mu^{p} p^{-\alpha}$, where, in the language of statistical physics, $\mu$ is a critical temperature and $\alpha$ a critical exponent. Amazingly, the theory of two-dimensional quantum gravity provides a conjecture for $\alpha$, based solely on the $\mathrm{O}(n)$ symmetry of the underlying matrix model, whereas the leading scaling exponent $\mu$ can only be accounted for in the exactly solvable cases. Comparing the conjecture for $\alpha(n)$ with our numerical results, we find good agreement in its expected range of validity $(0 \leq n \leq 2)$. 


\section{The matrix model and its renormalization}

The objects we want to consider are tangles with $2 k$ "external legs", that is roughly speaking the data of $k$ intervals embedded in a ball $B$ and whose endpoints are given distinct points on the boundary $\partial B$, plus an arbitrary number of (unoriented) circles embedded in $B$, all intertwined, and considered up to orientation preserving homeomorphisms of $B$ that reduce to the identity on $\partial B$. Tangles with 4 external legs will be simply called tangles. As mentioned in the introduction, we represent these objects using diagrams, that is regular projections on a plane, and restrict ourselves to alternating diagrams for which under- and over-passes alternate as one follows any connected component.

A natural framework is to introduce a matrix model which generates in its Feynman diagram expansion these link diagrams. We shall define it now.

\subsection{Definition of the $O(n)$ matrix model}

As in [11,8,10], we start with the following matrix integral over $N \times N$ hermitean matrices

$$
Z^{(N)}(n, g)=\int \prod_{a=1}^{n} \mathrm{~d} M_{a} \mathrm{e}^{N \operatorname{tr}\left(-\frac{1}{2} \sum_{a=1}^{n} M_{a}^{2}+\frac{g}{4} \sum_{a, b=1}^{n} M_{a} M_{b} M_{a} M_{b}\right)}
$$

where $n$ is a positive integer.

Expanding in power series in $g$ generates Feynman diagrams with double edges ("fat graph") drawn in $n$ colors in such a way that colors cross each other at the vertices. By taking the large $N$ limit one selects the planar diagrams, which can be redrawn as alternating link diagrams, cf Fig. 1.

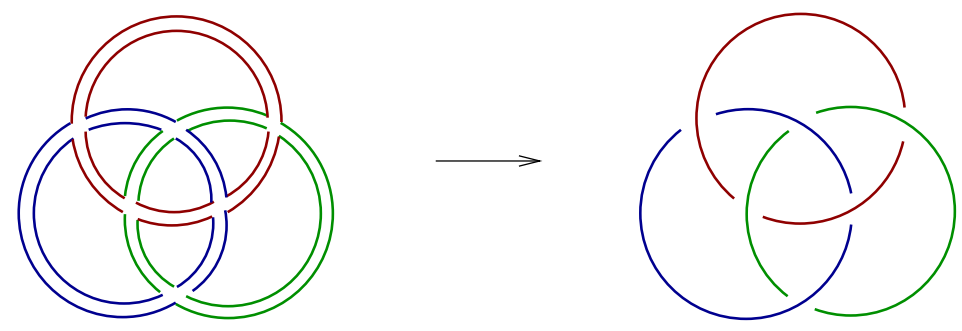

Fig. 1: A planar Feynman diagram of $(2.1)$ and the corresponding alternating link diagram. 
More precisely, the large $N$ "free energy"

$$
F(n, g)=\lim _{N \rightarrow \infty} \frac{\log Z^{(N)}(n, g)}{N^{2}}
$$

is a double generating function of the number $f_{k ; p}$ of alternating link diagrams with $k$ connected components and $p$ crossings (weighted by the inverse of their symmetry factor, and with mirror images identified):

$$
F(n, g)=\sum_{k=1}^{\infty} \sum_{p=1}^{\infty} f_{k ; p} n^{k} g^{p}
$$

If one is interested in counting objects with a weight of 1 , one cannot consider the free energy which corresponds to closed diagrams, but instead correlation functions of the model which generate diagrams with external legs, that is tangle diagrams. Typically, we shall be interested in the two-point function

$$
G(n, g) \equiv \lim _{N \rightarrow \infty}\left\langle\frac{1}{N} \operatorname{tr} M_{a}^{2}\right\rangle
$$

where the measure on the matrices $M_{a}$ is given by Eq. 2.1) and $a$ is any fixed index, which generates tangle diagrams with two external legs; and the connected four-point functions

$$
\begin{aligned}
& \Gamma_{1}(n, g)=\lim _{N \rightarrow \infty}\left\langle\frac{1}{N} \operatorname{tr}\left(M_{a} M_{b}\right)^{2}\right\rangle \\
& \Gamma_{2}(n, g)=\lim _{N \rightarrow \infty}\left\langle\frac{1}{N} \operatorname{tr}\left(M_{a}^{2} M_{b}^{2}\right)\right\rangle-G(n, g)^{2}
\end{aligned}
$$

where $a$ and $b$ are two distinct indices, which generate tangle diagrams with four external legs of type 1 and 2 (see Fig. 2).

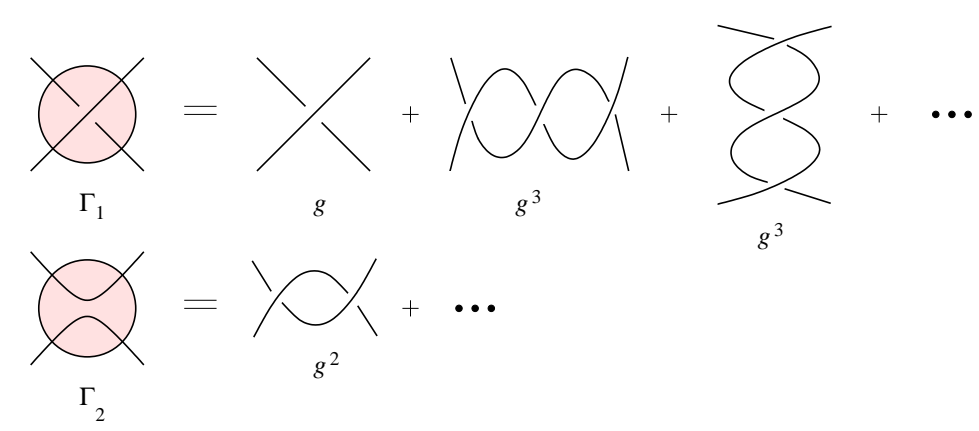

Fig. 2: Tangles of types 1 and 2 . 


\subsection{Renormalization of the $O(n)$ model}

The model presented above counts tangle diagrams, but not tangles. There is a redundancy in the counting since to a given tangle/link corresponds many diagrams. In order to properly count tangles, this redundancy must be removed. In the case of alternating diagrams one can distinguish two steps. First one must find a way to select only reduced diagrams which contain no irrelevant crossings (Fig. 3 a)); such diagrams will have minimum number of crossings. It turns out to be convenient to introduce at this point a closely related notion: a link is said to be prime if it cannot be decomposed into two pieces in the way depicted on Fig. 3 b). It is clear that at the level of diagrams, forbidding decompositions of the type of Fig. 3 b) automatically implies that the diagram is reduced; and we shall therefore restrict ourselves to prime links and tangles.

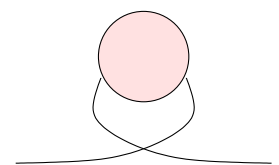

a)

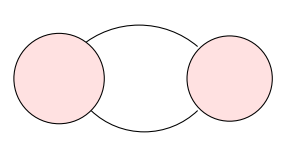

b)

Fig. 3: a) An irrelevant crossing. b) A non-prime link.

There may still be several reduced diagrams corresponding to the same link: according to the flyping conjecture, proved in [7], two such diagrams are related by a finite sequence of flypes, see Fig. 4.

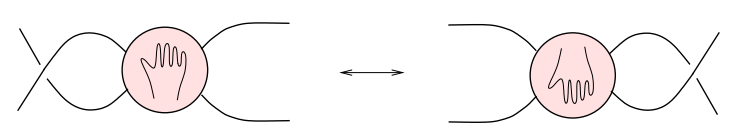

Fig. 4: A flype.

To summarize, there are two problems: a) the diagrams generated by applying Feynman rules are not necessarily reduced or prime; b) several reduced diagrams may correspond to the same knot due to the flyping equivalence. Following the study of [10], we note (cf Figs. 3 and 4) that this "overcounting" is local in the diagrams in the sense that problem a) is related to the existence of sub-diagrams with 2 external legs, whereas problem b) is related to a certain class of sub-diagrams with 4 external legs. Clearly such graphs can be cancelled by the inclusion of appropriate counterterms in the action. We are therefore led to the conclusion that we must renormalize the quadratic and quartic interactions of (2.1). Renormalization theory tell us that we should include in the action 
from the start every term compatible with the symmetries of the model, since they will be generated dynamically by the renormalization. A key observation is that, while there is only one quadratic $O(n)$-invariant term, there are two quartic $O(n)$-invariant terms, which leads to a generalized model with 3 coupling constants in the action:

$Z^{(N)}\left(n, t, g_{1}, g_{2}\right)=\int \prod_{a=1}^{n} \mathrm{~d} M_{a} \mathrm{e}^{N \operatorname{tr}\left(-\frac{t}{2} \sum_{a=1}^{n} M_{a}^{2}+\frac{g_{1}}{4} \sum_{a, b=1}^{n}\left(M_{a} M_{b}\right)^{2}+\frac{g_{2}}{2} \sum_{a, b=1}^{n} M_{a}^{2} M_{b}^{2}\right)}$

The Feynman rules of this model now allow loops of different colors to "avoid" each other, which one can imagine as tangencies (Fig. 5).

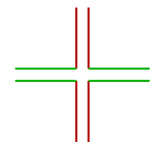

$g_{1}$

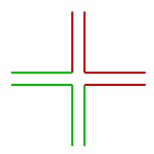

$g_{2}$

Fig. 5: Vertices of the generalized $O(n)$ matrix model.

We define again the correlation functions $G\left(n, t, g_{1}, g_{2}\right)$ and $\Gamma_{i}\left(n, t, g_{1}, g_{2}\right)$ (Eqs. (2.4) and (2.5)), and want to extract from them the counting of colored alternating tangles with external legs.

The idea is to find the expressions of $t(g), g_{1}(g)$ and $g_{2}(g)$ as a function of the renormalized coupling constant $g$, in such a way that the overcounting is suppressed and the correlation functions are generating series in $g$ (and $n$ ) of the number of colored tangles.

The derivation of the renormalization equations was performed in [10]. Consider first the removal of irrelevant crossings and non-prime links. It is clear that one must remove all two-legged subdiagrams, that is impose

$$
G\left(n, t, g_{1}, g_{2}\right)=1
$$

This implicitly fixes $t(g)$.

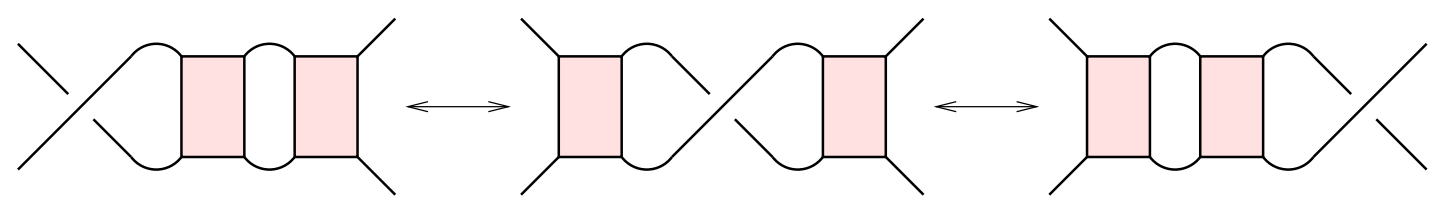

Fig. 6: Breaking a flype into two elementary flypes. 
Next, consider the flyping equivalence. A flype can be made of several "elementary" flypes (Fig. 6), an elementary flype being by definition one that cannot be decomposed any more in this way. In the terminology of QFT, these elementary flypes are one simple vertex connected by two edges to a non-trivial two-particle irreducible in the horizontal channel tangle diagram (H-2PI). We need to introduce auxiliary generating functions $H_{1}^{\prime}(g), H_{2}^{\prime}(g)$ and $V_{2}^{\prime}(g)$ for non-trivial H-2PI tangles of type 1 , of type 2 and of type 2 rotated by $\pi / 2$ respectively. By considering all possible insertions of elementary flypes as tangle subdiagrams of a diagram, we find (Fig. 7) that the renormalization of $g_{1}$ and $g_{2}$ is given by:

$$
\begin{aligned}
& g_{1}(g)=g\left(1-2 H_{2}^{\prime}(g)\right) \\
& g_{2}(g)=-g\left(H_{1}^{\prime}(g)+V_{2}^{\prime}(g)\right)
\end{aligned}
$$

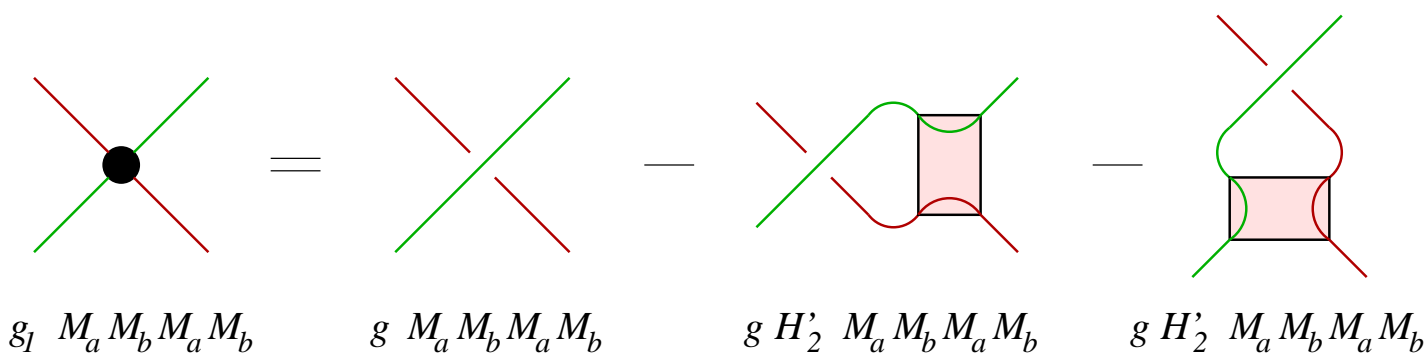

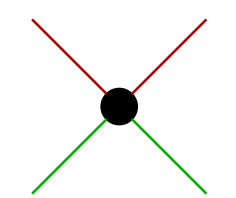

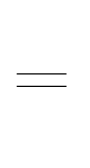

$g_{2} M_{a} M_{a} M_{b} M_{b}$

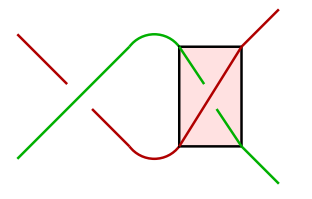

$g H_{l}^{\prime} M_{a} M_{a} M_{b} M_{b}$

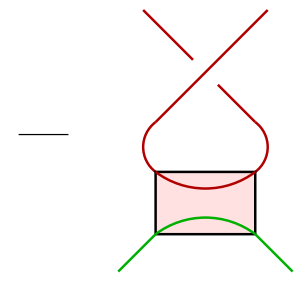

$g V_{2}^{\prime} M_{a} M_{a} M_{b} M_{b}$

Fig. 7: Counterterms needed to cancel flypes.

All that is left is to find the expressions of the auxiliary generating functions in terms of known quantities. They are easily obtained by decomposing the four-point functions in the horizontal and vertical channels [8]:

$$
\begin{aligned}
H_{2}^{\prime} \pm H_{1}^{\prime} & =1-\frac{1}{(1 \mp g)\left(1+\Gamma_{2} \pm \Gamma_{1}\right)} \\
H_{2}^{\prime}+n V_{2}^{\prime}+H_{1}^{\prime} & =1-\frac{1}{(1-g)\left(1+(n+1) \Gamma_{2}+\Gamma_{1}\right)}
\end{aligned}
$$


The three renormalization equations (2.7) and (2.8), supplemented by the expressions (2.9), are enough to fix $t(g), g_{1}(g)$ and $g_{2}(g)$. Solving them gives access to the $\Gamma_{i}$, which are the generating series of the numbers of prime alternating tangles of type $i$. However, we can go further. By computing other correlation functions in the model and composing them with the solutions $t(g), g_{1}(g), g_{2}(g)$ of the equations above, one can extract the generating functions of the number of alternating tangles with an arbitrary number of external legs. An example will be given below.

\section{An algorithm for the counting of decorated planar diagrams}

Unfortunately the matrix model described in section 2.1 cannot be solved for a generic value of $n$ (see however [12,8, 10] for solutions of particular values). We therefore turn to the description of an algorithm that allows to enumerate the required diagrams to a given order $p$ of crossings. This is one of several similar algorithms described in [13,9]. The numbers of diagrams thus computed are the input needed for the combinatorial treatment of section 2.2 .

The principle of the algorithm is to iterate a transfer matrix which "builds" the diagrams slice by slice: starting from an initial state consisting of all external legs (two in the case under consideration), the system is time evolved through the addition of $p$ intersections, until an empty final state is obtained.

We shall first concentrate on the enumeration of (prime, alternating) tangle diagrams, which correspond to the "unrenormalized" model of section 2. Adding tangencies, which is needed to take into account the flyping equivalence, will be discussed in Section 3.2, since it is an elementary extension of the algorithm.

\subsection{The single-step algorithm}

The algorithm we want to describe, called "single-step algorithm" in [9], is best explained by an example. Let us consider the tangle diagram shown in Fig. 8. Let us start from an initial state given by the two external legs (edges A and G). We want to "evolve" the various open lines by following them and adding the new crossings they meet.

Moving along either of the edges A or G, a new line segment (DE resp. HI) is encountered. The question then arises which of these to process first. We resolve this ambiguity by stipulating that in any given state, we evolve the line which at that instant is uppermost.

At time $t=1$, the edge $\mathrm{A}$ thus becomes $\mathrm{B}$, and the new line segment DE is added. The 


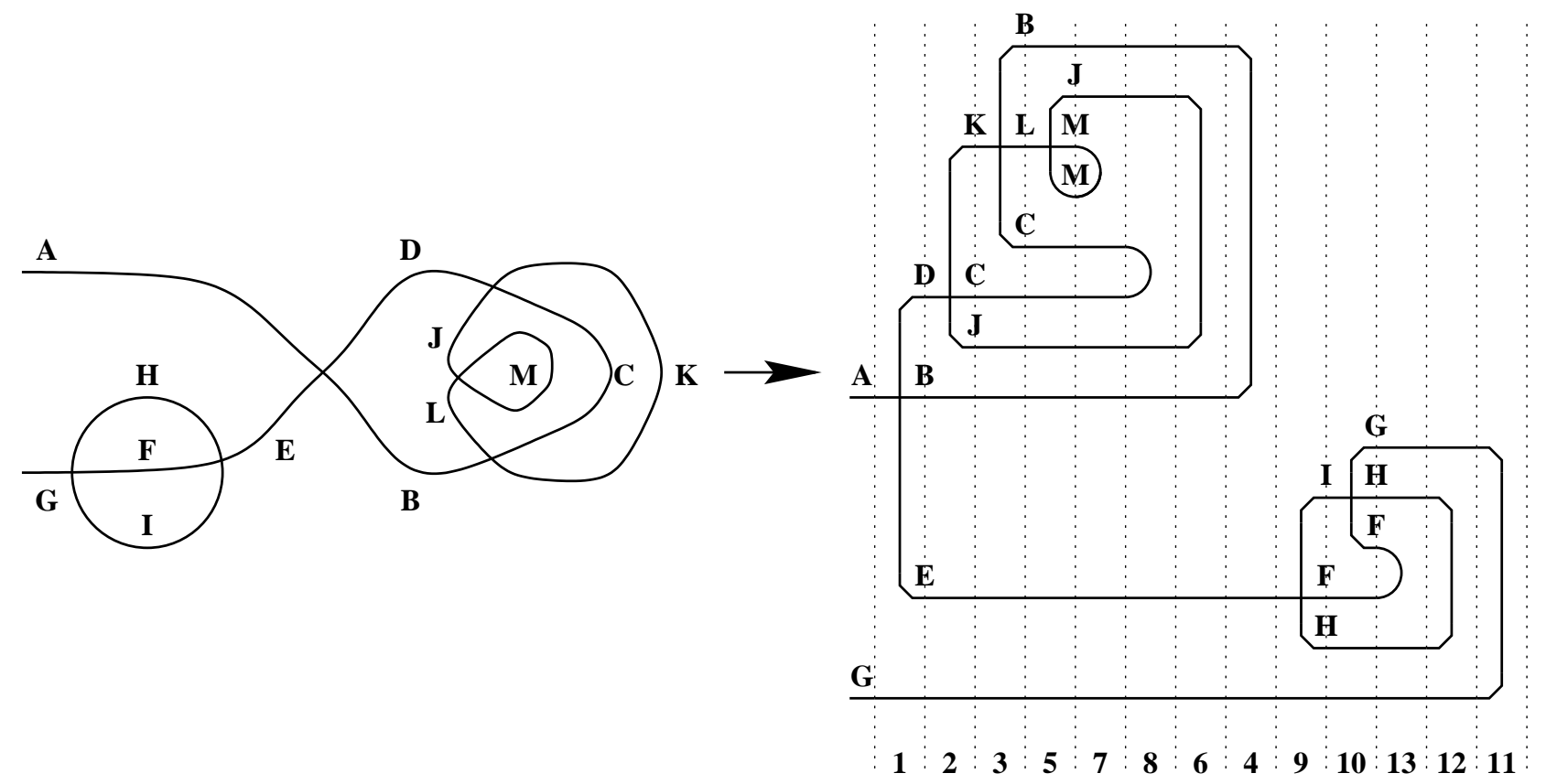

Fig. 8: Working principle of the single-step algorithm. a) A two-legged knot diagram with $p=6$ intersections and $k=3$ connected components. The edges are labelled from A to M. b) The same diagram in the timeslice representation. For reasons of clarity, the time slices are not drawn in chronological order.

edge $\mathrm{D}$ is now the new top line. Analogously, at the instants $t=2$ and $t=3$, the top line (D resp. K) crosses a new line segment, which is then added to the current state. We can formalize this by stating the transformation rule shown in Fig. 9.1.

At time $t=3$, the new top line carries the label $\mathrm{B}$, which was however already produced by the transformation acting at $t=1$. We therefore proceed, at $t=4$, to the identification of the two "copies" of B, joining them through an arch. This is an example of the general transformation rule shown in Fig. 9.2. The addition of an arch means that the lines intermediate between the two instances of $\mathrm{B}$ (at positions $p=1$ and $q$ on Fig. 9.2) can henceforth not communicate with the lines at the exterior of the arch. These "trapped lines" must therefore eventually evolve to the empty state (vacuum), independently of the rest of the diagram. Since the current top line must always be treated first, the evolution of a possible set of "trapped lines" must take place at a later time. Thus, on Fig. 8, we cannot always draw the time-slices in chronological order. The existence of a number of trapped lines is visualized on Fig. 9.2 by a delimiter (shown as a gray rectangle), which separated the remaining lines into two blocks. Lines in different blocks cannot communicate, and so the transformation 2) only applies when $p$ and $q$ belong to the same block. 
1)

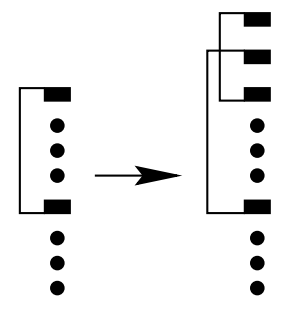

2)

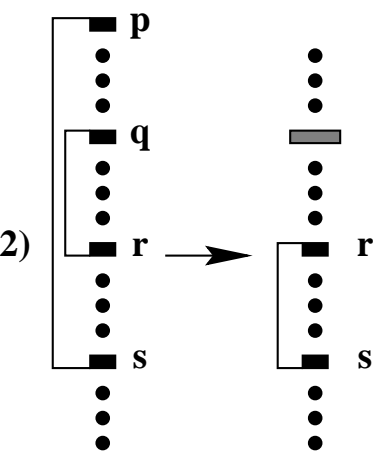

Fig. 9: The two types of transformations in the single-step algorithm. 1) Addition of a new line segment. 2) Identification of the top line (at position $p$ ) with another line (at position $q$ ), accompanied with the creation of a new block.

The purpose of the transfer matrix is not only to count the total number of tangle diagrams, but to do so for any fixed number of connected components. In particular, when performing a type 2 transformation, we need to know whether the points $p$ and $q$ were already connected though an arbitrary number of edges at an earlier time. On Fig. 9 we have represented this information by a number of lines on the left, connecting the points at a given instant into pairs. It may thus happen that on Fig. $9.2, r=p$ and $s=q$. In this case, the type 2 transformation marks the completion of one connected component in the tangle diagram.

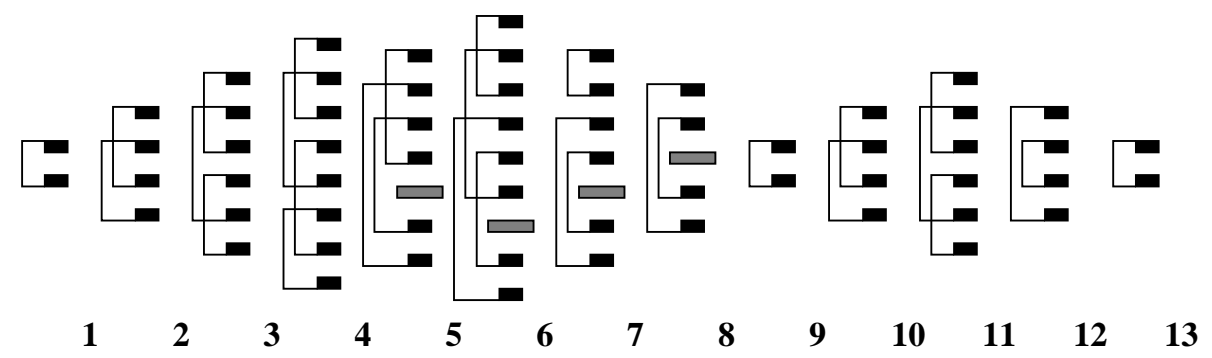

Fig. 10: Intermediate states produced by applying the single-step algorithm to the tangle diagram shown in Fig. 8.

We are now ready to define the set of states on which the transfer matrix acts. A state is defined by an even number of points (represented on Fig. 9 as black rectangles), connected into pairs by means of the edges encountered at previous times. In addition, the points are divided into $\ell+1$ blocks by means of $\ell \geq 0$ delimiters. On Fig. 10 we show the set of intermediate states corresponding to the time-slice representation of the tangle of Fig. 8. 
Finally, we must define the transfer matrix $T$ which counts all tangle diagrams with $\tilde{p}$ vertices and $k$ connected components. Its entries $T_{a b}$, where $a$ and $b$ are two basis states of the kind just defined, are 0 unless $b$ is a descendant of $a$. An allowed state $b$ is a descendant of $a$ if it can be obtained via a transformation of one of the two types shown on Fig. 9 (for an arbitrary even $q \geq 2$ belonging to the same block as $p=1$ ), followed by an arbitrary number of simplifications. $T_{a b}$ is then the sum over all transformations from $a$ to $b$ of the corresponding weight: 1 or $n$ depending on whether one closes a connected component or not. The simplications are elimination of superfluous delimiters, and reduction of states to a "normal form" by using various symmetries of the transfer matrix [9].

\subsection{Tangencies}

Until now we have been discussing the enumeration of tangle diagrams in which every vertex represents a crossing. However, to account for the flype equivalence we need to enumerate more general diagrams with $p_{1}$ intersections and $p_{2}$ tangencies, as discussed in Section 2. Adding a tangency rather than an intersection is obtained by modifying the transformation if Fig. 9.1, so that the two points added at time $t+1$ are both immediately above (resp. immediately below) the uppermost point at time $t$. any given time, specifies how many tangency transformations were used prior to that instant. The desired diagrams are then generated by sequences of $p_{1}$ crossing transformations (type 1), $p_{2}$ trangency transformations, and $p_{1}+p_{2}+1$ transformations of type 2 , so that no intermediate state is empty.

\section{Numerical results and analysis}

The implementation of the above algorithm provides an efficient numerical way to count the diagrams needed for our purposes. We present here some of the data obtained in $[13,9]$.

First, we give one example of the "raw data" obtained from the algorithm. It corresponds to counting a certain set of diagrams without taking into account topological equivalences: in the present case, connected two-legged alternating diagrams (called twolegged knot diagrams in [13]), see Tab. 1. The 2PI diagrams are precisely the reduced diagrams of prime tangles. The importance of going to orders as high as possible comes from the issue of the asymptotic behavior which will be discussed below. 


\begin{tabular}{c|cc}
$p$ & $G$ & $\Sigma_{2}$ \\
\hline 0 & 1 & 0 \\
1 & 2 & 2 \\
2 & 8 & 0 \\
3 & 42 & 2 \\
4 & 260 & 4 \\
5 & 1796 & 60 \\
6 & 13396 & 226 \\
7 & 105706 & 1076 \\
8 & 870772 & 5156 \\
9 & 7420836 & 24984 \\
10 & 65004584 & 128548 \\
11 & 582521748 & 663040 \\
12 & 5320936416 & 3514968 \\
13 & 49402687392 & 18918792 \\
14 & 465189744448 & 103123906 \\
15 & 4434492302426 & 569877652 \\
16 & 42731740126228 & 3180066004 \\
17 & 415736458808868 & 17921451960 \\
18 & 4079436831493480 & 101842206548 \\
19 & 40338413922226212 & 583109887600 \\
20 & 401652846850965808 & 194992266688816 \\
21 & 4024556509468827432 & 3361640932872 \\
22 & 40558226664529024000 &
\end{tabular}

Tab. 1: Total number $G$ of two-legged diagrams, as well as the subset $\Sigma_{2}$ of 2PI diagrams.

Let us also give two examples of renormalized data. Tab. 2 shows the number of fourlegged prime alternating tangles of the two possible types (Fig. 2) with arbitrary number of connected components, while Tab. 3 shows the number of six-legged tangles, which exist in five types (Fig. 11).

Finally, let us discuss the expected asymptotic behavior of these series. Let us consider an unrenormalized generating series for tangle diagrams, say $G(n, g)=\sum_{p=1}^{\infty} a_{p}(n) g^{p}$. Then it is expected that

$$
a_{p}(n) \stackrel{p \rightarrow \infty}{\approx} \mathrm{e}^{\hat{s}(n) p} p^{-\alpha(n)}
$$

The conjecture holds for any correlation function in the model; for the free energy $F(n, g)=$ $\sum_{p=1}^{\infty} f_{p}(n) g^{p}$, that is the generating series for link diagrams (the symmetry factors being 


\begin{tabular}{|c|c|c|c|c|c|c|c|c|c|c|c|c|}
\hline \multirow[b]{2}{*}{$p^{k}$} & \multicolumn{6}{|c|}{$\Gamma_{1}$} & \multicolumn{6}{|c|}{$\Gamma_{2}$} \\
\hline & 0 & 1 & 2 & 3 & 4 & 56 & 0 & 1 & 2 & 3 & 4 & 56 \\
\hline 1 & 1 & & & & & & 0 & & & & & \\
\hline 2 & 0 & & & & & & 1 & & & & & \\
\hline 3 & 2 & & & & & & 1 & & & & & \\
\hline 4 & 2 & & & & & & 3 & 1 & & & & \\
\hline 5 & 6 & 3 & & & & & 9 & 1 & & & & \\
\hline 6 & 30 & 2 & & & & & 21 & 11 & 1 & & & \\
\hline 7 & 62 & 40 & 2 & & & & 101 & 32 & 1 & & & \\
\hline 8 & 382 & 106 & 2 & & & & 346 & 153 & 24 & 1 & & \\
\hline 9 & 1338 & 548 & 83 & 2 & & & 1576 & 747 & 68 & 1 & & \\
\hline 10 & 6216 & 2968 & 194 & 2 & & & 7040 & 3162 & 562 & 43 & 1 & \\
\hline 11 & 29656 & 11966 & 2160 & 124 & 2 & & 31556 & 17188 & 2671 & 121 & 1 & \\
\hline 12 & 131316 & 71422 & 9554 & 316 & 2 & & 153916 & 80490 & 15295 & 1484 & 69 & 1 \\
\hline 13 & 669138 & 328376 & 58985 & 5189 & 184 & 2 & 724758 & 425381 & 87865 & 6991 & 194 & 1 \\
\hline 14 & 3156172 & 1796974 & 347038 & 22454 & 478 & 2 & 3610768 & 2176099 & 471620 & 52231 & 3280 & 1031 \\
\hline 15 & 16032652 & 9298054 & 1864884 & 193658 & 10428 & 2602 & 17853814 & 11376072 & 2768255 & 308697 & 15431 & 2901 \\
\hline
\end{tabular}

Tab. 2: Table of the number of prime alternating tangles with 4 external legs.

\begin{tabular}{|c|c|c|c|c|c|c|c|c|c|c|c|c|c|c|c|c|c|c|c|}
\hline \multirow[b]{2}{*}{$p^{k}$} & \multicolumn{4}{|c|}{$\Xi_{1}$} & \multicolumn{3}{|c|}{$\Xi_{2}$} & \multicolumn{4}{|c|}{$\Xi_{3}$} & \multicolumn{4}{|c|}{$\Xi_{4}$} & \multicolumn{4}{|c|}{$\Xi_{5}$} \\
\hline & 0 & 1 & 2 & 3 & 0 & 1 & 23 & 0 & 1 & 2 & 34 & 0 & 1 & 2 & 3 & 0 & 1 & 2 & 3 \\
\hline 2 & 0 & & & & 1 & & & 0 & & & & 0 & & & & 0 & & & \\
\hline 3 & 2 & & & & 0 & & & 2 & & & & 0 & & & & 0 & & & \\
\hline 4 & 0 & & & & 7 & & & 2 & & & & 4 & & & & 3 & & & \\
\hline 5 & 18 & & & & 6 & & & 16 & 2 & & & 8 & & & & 9 & & & \\
\hline 6 & 18 & & & & 53 & 8 & & 42 & 2 & & & 42 & 7 & & & 41 & 7 & & \\
\hline 7 & 156 & 24 & & & 154 & 6 & & 171 & 44 & 2 & & 156 & 14 & & & 168 & 21 & & \\
\hline 8 & 516 & 18 & & & 609 & 181 & 6 & 748 & 114 & 2 & & 608 & 153 & 10 & & 663 & 165 & 12 & \\
\hline 9 & 2016 & 598 & 18 & & 2956 & 422 & 6 & 2877 & 858 & 81 & 2 & 2850 & 586 & 20 & & 3072 & 740 & 36 & \\
\hline 10 & 10608 & 1428 & 18 & & 11203 & 3498 & 3186 & 14037 & 3752 & 213 & 2 & 11918 & 3445 & 364 & 13 & 13347 & 3966 & 438 & 18 \\
\hline 11 & 40428 & 12318 & 10621 & & 57664 & 15330 & 7386 & 61028 & 19757 & 2511 & 1312 & 57602 & 17558 & 1406 & 26 & 63393 & 20994 & 2040 & 54 \\
\hline
\end{tabular}

Tab. 3: Table of the number of prime alternating tangles with 6 external legs.

most likely irrelevant for the asymptotic behavior), it is

$$
f_{p}(n) \stackrel{p \rightarrow \infty}{\approx} \mathrm{e}^{\hat{s}(n) p} p^{-\alpha(n)-1}
$$




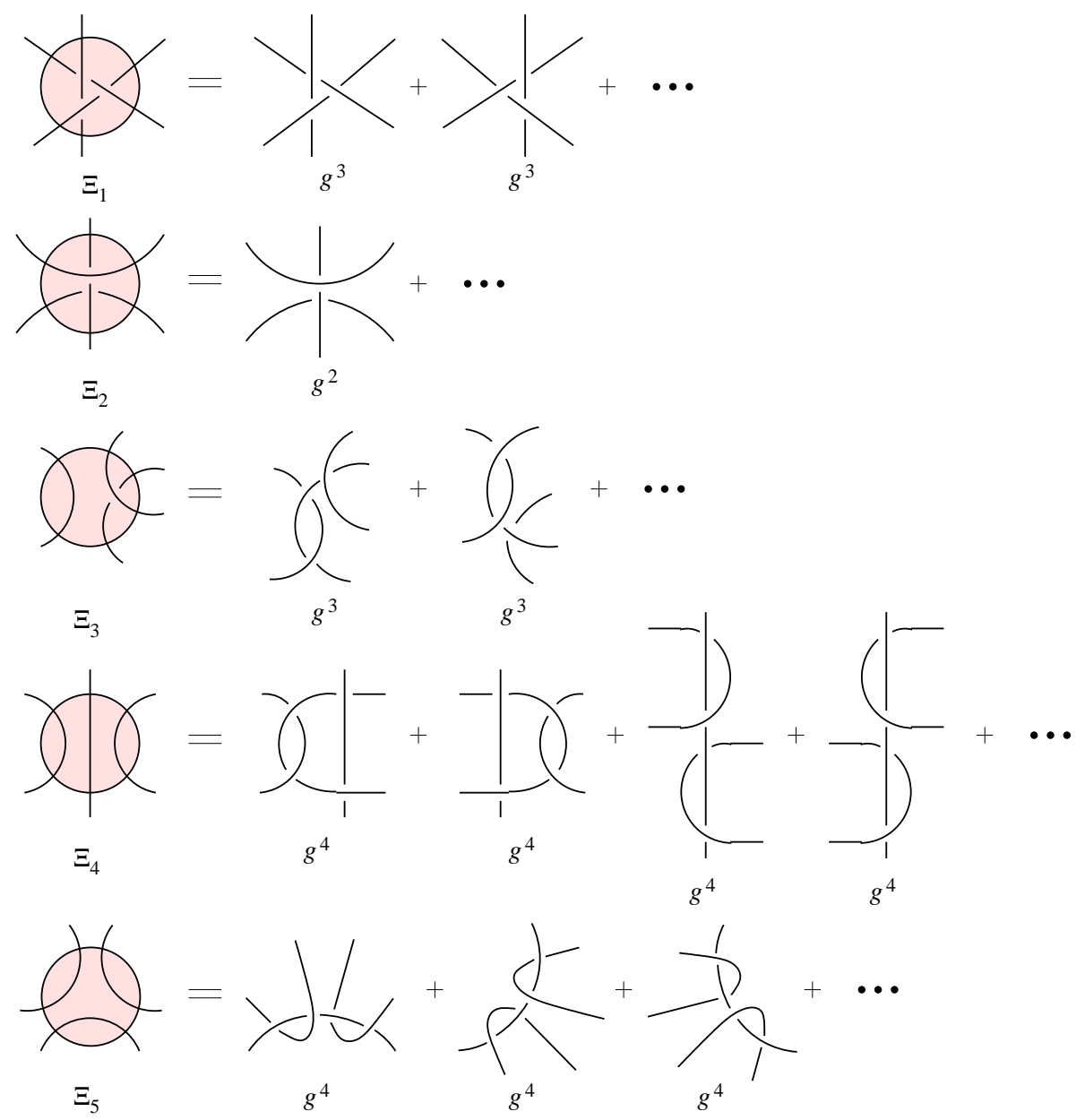

Fig. 11: The five types of tangles with 6 external legs.

The bulk entropy $\hat{s}(n)$ can be easily extracted with a good accuracy from the numerical data. However it is non universal, which implies that it is not preserved by the renormalization of the model. On the other hand, the exponent $\alpha(n)$ is universal, and presumably preserved by the renormalization (unless an unusual phase transition takes place in the model). Since the raw data are "cleaner" than the renormalized data, they are better suited to the extraction of $\alpha(n)$. A conjecture made in [11], based in particular on the KPZ relation [14], is that for $n$ analytically continued to $0 \leq n \leq 2$, one has

$$
\alpha(n=-2 \cos (\pi \nu))=1+1 / \nu \quad 1 / 2 \leq \nu \leq 1
$$

Using Tab. 1 one can try to evaluate $\alpha=\alpha(n=0)$, the exponent of tangles with minimum number of components, which also gives the exponent of knots according to Eq. (4.2). The conjecture (4.3) yields $\alpha=3$. Unfortunately, with the current data, it is difficult to estimate $\alpha$ without any knowledge of the subleading corrections. Indeed, a 
direct fit gives $\alpha \approx 2.76$ but with very weak accuracy. In [13], it was suggested to fit the data with $a_{p}=\mathrm{e}^{\hat{s} p} p^{-\alpha}(a \log p+b+o(1))$, the result being in good agreement with the conjecture:

$$
\mathrm{e}^{\hat{s}}=11.416 \pm 0.005 \quad \alpha=2.97 \pm 0.06 \quad a=0.04 \pm 0.02 \quad b=0.1 \pm 0.03
$$

However there is little evidence for such a logarithmic correction, and the issue remains open. A more detailed discussion of $\alpha(n)$ for all $n$ can be found in [9]. 


\section{References}

[1] J. Hoste, M. Thistlethwaite and J. Weeks, The First 1,701,936 Knots, The Mathematical Intelligencer 20 (1998) 33-48.

[2] J. Ozanam Récréations mathématiques et physiques (Paris, 1694, 2 vols.). [Revised by Montucla (Paris, 1778, 4 vols.); English translation by Hutton (London, 1803, 4 vols.)]. We here cite p. 222 , vol. 4 of the 1725 edition. We do not know whether the idea was already manifest in the books by C. Bachet (1612 and 1624), and by C. Mydorge (1630).

[3] G. W. von Leibniz, cited in Histoire de l'Académie royale des Sciences de Paris pour l'année 1771, p. 55.

[4] A.-T. Vandermonde, Remarques sur les problèmes de situation, Mém. de l'Ac. des Sc. de Paris pour l'année 1771, p. 566 (Paris, 1774).

[5] C. Sundberg and M. Thistlethwaite, The rate of Growth of the Number of Prime Alternating Links and Tangles, Pac. J. Math. 182 (1998) 329-358.

[6] L.H. Kauffman, Knots and Physics, World Scientific Pub Co (1994).

[7] W.W. Menasco and M.B. Thistlethwaite, The Tait Flyping Conjecture, Bull. Amer. Math. Soc. 25 (1991) 403-412; The Classification of Alternating Links, Ann. Math. 138 (1993) 113-171.

[8] P. Zinn-Justin and J.-B. Zuber, On the Counting of Colored Tangles, Journal of Knot Theory and its Ramifications 9 (2000) 1127-1141 (preprint math-ph/0002020).

[9] J. L. Jacobsen and P. Zinn-Justin, A Transfer Matrix approach to the Enumeration of Colored Links (preprint math-ph/0104009).

[10] P. Zinn-Justin, The General $O(n)$ Quartic Matrix Model and its Application to counting Tangles and Links (preprint math-ph/0106005).

[11] P. Zinn-Justin, Some Matrix Integrals related to Knots and Links, proceedings of the 1999 semester of the MSRI "Random Matrices and their Applications", MSRI Publications Vol. 40 (2001) (preprint math-ph/9910010).

[12] P. Zinn-Justin, J.-B. Zuber, Matrix Integrals and the Counting of Tangles and Links, proceedings of the 11th International Conference on Formal Power Series and Algebraic Combinatorics, Barcelona June 1999; to appear in Discrete Mathematics (preprint math-ph/9904019).

[13] J. L. Jacobsen and P. Zinn-Justin, A Transfer Matrix approach to the Enumeration of Knots (preprint math-ph/0102015).

[14] V. G. Knizhnik, A. M. Polyakov and A. B. Zamolodchikov, Fractal structure of $2 D$ quantum gravity, Mod. Phys. Lett. A 3, 819-826 (1988); F. David, Conformal field theories coupled to $2 D$ gravity in the conformal gauge, Mod. Phys. Lett. A 3, 16511656 (1988); J. Distler and H. Kawai, Conformal field theory and 2D quantum gravity, Nucl. Phys. B 321, 509 (1989). 